\title{
Understanding human diseases with high-throughput quantitative measurement and analysis of molecular signatures
}

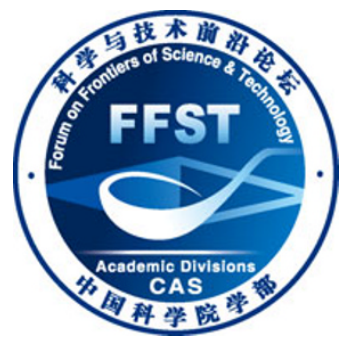

\author{
YANG Li, WEI Gang, TANG Kun, NARDINI Christine \& HAN Jing-Dong J. \\ CAS Key Laboratory of Computational Biology, CAS-MPG Institute for Computational Biology, Shanghai Institutes of Biological Sciences, Chinese \\ Academy of Sciences, Shanghai 200031, China
}

Received August 7, 2012; accepted November 19, 2012

\begin{abstract}
Microarray and deep sequencing technologies have provided unprecedented opportunities for mapping genome mutations, RNA transcripts, transcription factor binding, and histone modifications at high resolution at the genome-wide level. This has revolutionized the way in which transcriptomes, regulatory networks and epigenetic regulations have been studied and large amounts of heterogeneous data have been generated. Although efforts are being made to integrate these datasets unbiasedly and efficiently, how best to do this still remains a challenge. Here we review major impacts of high-throughput genome-wide data generation, their relevance to human diseases, and various bioinformatics approaches for data integration. Finally, we provide a case study on inflammatory diseases.
\end{abstract}

genomics, epigenomics, phenomics, integration, data analysis

Citation: Yang L, Wei G, Tang K, et al. Understanding human diseases with high-throughput quantitative measurement and analysis of molecular signatures. Sci China Life Sci, 2013, 56: 213-219, doi: 10.1007/s11427-013-4445-9

Twelve years ago, the unveiling of the first human reference genome sequence [1,2] inspired researchers to believe that genome-based discoveries would revolutionize the study and clinical treatment of human diseases. As genome sequences from different individuals became available, comparative genomics using computational approaches emerged as a powerful method for understanding gene functions at the genome-wide level. These approaches unveiled more variations between individuals than were initially expected [3]. Genomic variations (including single nucleotide polymorphism (SNPs) and insertions and deletions (indels)) responsible for some of hereditary diseases have been identified and applied to examine genomes of thousands of individuals for correlations between the presence of variants and traits of interests [4]. First microarrays were used, then exon sequencing, and now whole genome sequencing has become a popular tool [5,6]. Currently, many variations

*Corresponding author (email: jdhan@ @icb.ac.cn) from numerous sites in the genome have been successfully connected with different human diseases including various types of cancers [6] using DNA sequencing technology which underwent a 14000-fold drop in cost between 1999 and 2009 [7], and computational imputation methods [8].

Though most studies have focused on the connection between genomic variations (both common and rare) and human diseases, mechanisms underlying many of the DNA variations have not been clearly addressed. Genome information alone is not sufficient to interpret complex diseases [9]. Evidence at epigenome, post-transcriptome, and even the human microbiome levels is beginning to shed new light on human disease-related studies beyond the genome level.

\section{Epigenome and human diseases}

Epigenetics is commonly defined as the study of heritable changes in gene activity and expression without changes in 
the genomic sequence. Epigenetic mechanisms, which mainly consist of states and changes in DNA methylation, histone modification, non-coding RNAs (ncRNAs), and higher-order chromatin structure, are believed to play important roles in regulating genome functions such as gene transcription and genomic DNA replication [10]. Emerging lines of evidence indicate that epigenetic modifications significantly contribute to normal cellular developmental processes and aberrant epigenetic controls are linked to many types of diseases [11]. For example, epigenetic mechanisms have a profound role in cancer development. On the one hand, numerous changes in DNA methylation and histone modification accompanied by genomic mutations in many loci of the epigenetic regulator genes have been found in multiple types of cancers, indicating that epigenetic alterations contribute to cancer development [12]. On the other hand, epigenetic mechanisms provide novel targets for therapeutic intervention, exemplified by the use of histone deacetylase inhibitors (HDACi) to treat several types of cancers such as T-cell lymphoma [13]. In addition to its role in cancers, epigenetic dysregulation can lead to many immune and neuron disorders including systemic lupus erythematosus (SLE), rheumatoid arthritis, type 1 diabetes, Rett syndrome and Alzheimer's disease [14-18]. Although knowledge of epigenetic mechanisms in these diseases is growing fast, a more comprehensive understanding of epigenetic processes is still required for safe and efficient diagnosis and treatment of these diseases.

The first step to understand epigenetic mechanisms is to identify the epigenetic changes that contribute to cellular phenotypes in normal development and in diseases. Thanks to recent advances in next-generation sequencing (NGS) technologies, genome-wide maps of the epigenome can be generated at a much lower cost, thus providing landscapes of DNA methylation, histone modification and other chromatin features. In 2007, the first comprehensive epigenome map of human CD4 cells was drawn using a pioneering combination of chromatin immunoprecipitation followed by high throughput sequencing (ChIP-seq); this map includes 20 histone modifications as well as histone variant H2A.Z, RNA polymerase II, and insulator binding protein CTCF [19]. In 2008, the NIH Roadmap Epigenomics Mapping Consortium was launched with the aim of generating a public epigenomic data resource for biomedical research. In this project, multiple levels of epigenetic features in different cell types are to be mapped; for example, DNA methylation is assayed by bisulfite sequencing (BS-seq) which treats genomic DNA with sodium bisulfite before sequencing [20], histone modifications are mapped by ChIP-seq as mentioned above, and chromatin accessibility is evaluated by sequencing DNase I hypersensitive sites [21].

Although these novel genome-wide approaches can generate epigenome data sets at different levels, challenges are still to integrate them with the transcriptome (all RNA transcripts), to discover epigenomic signatures in a specific cell type or disease, and to identify important epigenetic regulators that contribute to normal and disease developmental processes. In addition, huge amounts of epigenome data are generated by high-throughput sequencing, which requires the development of novel computational tools to analyze it. For example, $\mathrm{Yu}$ et al. [22] have developed a Bayesian network model to infer combinational interactions of various histone modifications and their effect on transcriptional regulation. In addition, using comparative epigenomics from both DNA and histone modifications can further reveal regulatory features of the genome [23].

Epigenomes and combined integrative computational analyses have also revealed some of the crucial regulatory roles that epigenetic modifications play in many human disease-related processes such as aging [24-26] and T-cell maturation $[27,28]$.

\section{Transcriptome and human diseases}

The transcriptome in a given organism/cell can be very different between individuals, tissues, cell-types, and developmental stages. The recent advent of high-resolution tiling arrays and next-generation deep sequencing technologies have changed the way in which transcriptomes are studied, and also made it possible to link transcriptome changes with human diseases at the single nucleotide resolution level. For example, whole transcriptome analyses have demonstrated consistent differences between the normal and autistic human brain, and provided further evidence of transcriptional and splicing dysregulation as underlying mechanisms of neuronal dysfunction in autism [29]. Another large-scale spatio-temporal transcriptome analysis of human brains provided a comprehensive data set of the human brain transcriptome as well as insights into the transcriptional foundations of human neurodevelopment and neurobiological diseases [30]. In addition, using a different enrichment method, Chen and colleagues uncovered a number of long noncoding RNAs (lncRNA) from introns [31]. Interestingly, further analyses showed that some of these IncRNA were capped with small nucleolar RNAs (snoRNAs) at both ends and were abundantly expressed from the imprinted region on chromosome 15 that has been implicated in human PraderWilli syndrome [32]. Some preliminary evidence in both human and mouse has indicated the possible role of the microbiome in diabetes, obesity, and liver function, though in-depth clinical studies are still being carried out [33]. When "omic" datasets are combined, a much broader view of the mechanism underlying diseases is expected as a result [34]. Chen and colleagues showcased an integrative personal omics profile consisting of genomic, transcriptomic, proteomic, metabolomic, and autoantibody profiles from a single individual over a 14-month period, which revealed extensive and dynamic changes across healthy and diseased conditions, and uncovered both DNA variation and an un- 
expected post-transcriptional mechanism during healthy and diseased states [35].

\section{Digital and quantitative phenome and human diseases}

While novel sequencing technologies are rapidly expanding the borders of genomics, methodologies for collecting phenotype data are also facing major transitions. Large-scale high-throughput phenotyping is becoming increasingly important in the post-genomics era. A comprehensive set of phenotypic traits collected at cell, tissue, organ or organism level is defined as a phenome [36]. Thus, phenomics can be defined as the study of the phenome as well as how it is determined or affected by, aside from the environment, the other omics data including but not limited to the genome, transcriptome, and proteome.

A central interest of phenomic research is the study of morphology and appearance traits. The overall morphology and appearance of an organism results from coordinated biological actions at all levels. Phenotypes provide rich information for many research areas such as evolution, development and human disease studies. In large-scale genetic modification studies in model organisms, morphological changes are main pathological evidences of targeted mutations [37-39]. The soft tissue of the human face is a complex geometric surface composed of many important organs, including eyes, nose, ears, and mouth. Morphogenesis of the craniofacial structure is a sophisticated development process that involves, to name a few, neural tube closure, midline patterning, neural crest generation and migration, outgrowth, patterning, and differentiation of the facial primordia and the branchial arches [40]. All of these processes are under the elaborate control of many signaling pathways such as SHH, FGF and BMP pathways [41]. Given its essential biological functions and structural complexity, the human face can tell a great deal about an individual's health conditions, from genetic defects to common diseases, and from aging to mental health. Rare genetic disorders in many syndromes cause characteristic abnormal facial features; for example, Down syndrome [42], Rubinstein-Taybi syndrome [43], Sotos syndrome [44] and Noonan syndrome [45]. More generally, common genetic variants were found associated with higher risks of facial dysmorphisms such as cleft palate and cleft lip [46-49]. Interestingly, some cleft lip risk alleles were found associated with facial morphological deviations in parents of patients as well as in normal individuals [50], suggesting the potential use of face examination in disease risk evaluation. In fact, traditional Chinese medicine has long used face examination for general disease diagnosis. Apart from disease research, the human face has been intensively studied in a wide range of medical fields such as forensics [51], psychology [52,53] and aging [54, 55].

Novel technologies and algorithms are being developed to either capture comprehensive phenome data or to extract specific physiological information from the human face. Quick face recognition algorithms have for a long time been deployed mainly in security surveillance [56,57]. Recently, weak signals extracted from video data were used to measure cardiac pulse from human face [58]. The introduction of high resolution three-dimensional (3D) image acquiring technologies such as the $3 \mathrm{dMDface}{ }^{\circledR}$ system (www.3dmd. com), enabled the collection of complete facial shape and texture data at the phenome level. Nonetheless, most current studies use only a small fraction of such data, usually a set of landmarks and/or their mutual distances and angles [59-61]. This has largely constrained the power of technologies to associate facial patterns with specific diseases, because the pathological phenotype is usually the sum of subtle curvature and pigmentation changes over the entire facial surface. Nonetheless, methods have been developed to register the 3D facial images by their dense surface meshes (3D dense surface registration, 3D-DSR), which allow the thousands of 3D pixel points for each of sample faces to be anatomically aligned across genders, age groups, ethnicities as well as disease/control groups [62-64]. Inferences and analyses can therefore be carried out on the face phenome data in a fully quantitative way that is analogous to how the genome data is used. Recently, Guo et al. [65] incorporated a novel algorithm of accurate 3D face landmarking into the registration method, which achieved complete automation of 3D-DSR at high throughput and robustness. 3D-DSR methods have been used to calculate average faces for different ethnicities, genders and various syndromes [66-68], and to fit trajectories of face growth over age [69]. More importantly, facial signatures of many genetic disorders were extracted using 3D-DSR, rendering unprecedented power and efficiency to automatic disease discrimination [70]. Similar methods have also been tested for complex diseases such as autisms and epilepsy [71,72]. Unfortunately, as most of these disease studies were carried out in $\mathrm{Eu}-$ ropean populations, results cannot be directly applied to other ethnic groups because of the obvious divergence of basal facial shapes and genomic backgrounds. Peng et al. first revealed that, in a Han Chinese population, a locus that was associated with non-syndromic cleft lip was also associated with common facial morphological variations in healthy individuals (unpublished data). In the long run, subtle characteristic features may be extracted not only to diagnose rare genetic disorders but also to manage general health conditions, such as common disorders like skin aging and stress. Given the non-invasive nature of face imaging technologies, advances in this field may strongly promote applications of personalized medicine. 


\section{Integrative analyses of heterogeneous high- throughput data}

High-throughput data is increasing rapidly at the molecular level by microarrays and deep sequencing and at the phenotypic level by digitized and quantitative imaging. There is an urgent need to develop approaches that can efficiently integrate different data sets in an unbiased manner. Methods ranging from simple correlation based network analysis to more sophisticated Bayesian network analysis have all been elicited to address this need [73]. New algorithms have also been developed to specifically address the problem of integrating different layers of data. For example, flow optimization algorithms were used to identify main pathways from protein-protein interaction networks that link genetic screen hits to gene expression changes involved in neurodegeneration [74], microRNA (miRNA) expression changes and mRNA expression changes in response to extremely high altitudes [75], or Genome-Wide Association Study (GWAS) hits to differentially expressed genes between control and disease cohorts [76]. These innovative integration approaches for different types of data from different layers have the potential to identify global regulation patterns, and also to provide detailed biological insights regarding complex biological processes, such as the development of complex phenotypes and diseases (Figure 1).

\section{A case study: application to immune diseases}

As discussed above, unprecedented insights that have been revealed using sequencing technologies are almost countless. One of high impact areas for translational medicine is related to the study of autoimmune diseases, which affect populations in unexpected proportions (2.5 times more than cancer and slightly more than heart diseases; American $\mathrm{Au}$

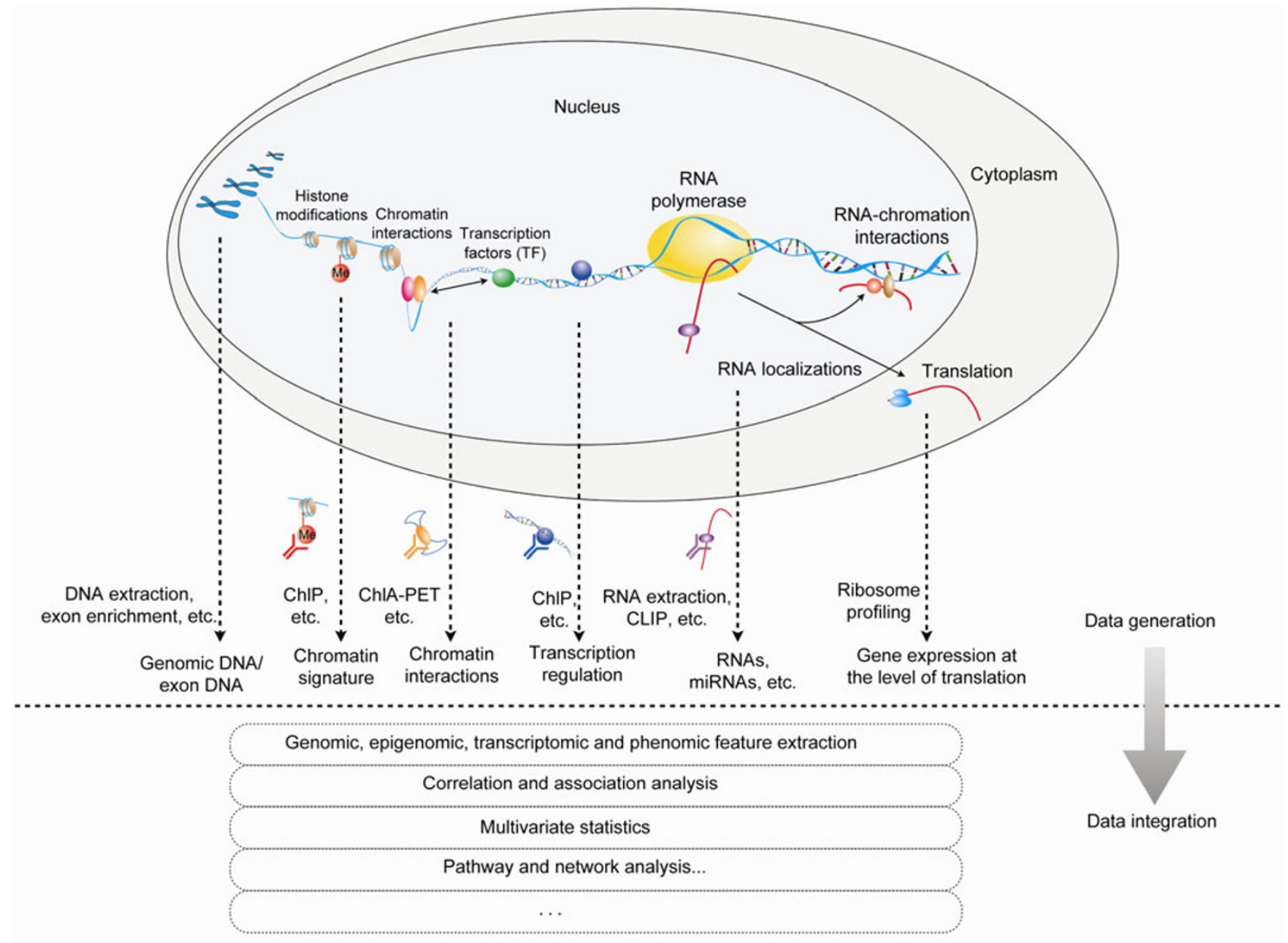

Figure 1 Strategy for data generation and integration of multi-layer high-throughput data sets. By generating and computationally integrating highthroughput data at various systems levels, a comprehensive atlas of detailed molecular signatures for complex human diseases can be depicted. The atlas can then be used to develop systematic explanations of clinical practices and diagnosis for complex diseases, thus laying foundations for the development of personalized medicine in the future. Relevant experimental methods for interrogating functional genomic elements and other biomolecules and the major downstream analysis approaches are listed (see text for details). ChIP, chromatin immuno-precipitation; ChIA-PET, chromatin interaction analysis with paired-end tag sequencing. 
toimmune Related Diseases Association, Inc. (AARDA) statistics (http://www.aarda.org/autoimmune_statistics.php)). These maladies often affect patients during their productive age.

Rheumatoid arthritis (RA) is often used as a model disease for autoimmune diseases. RA is a disabling disease with a still unknown etiology that attacks the synovial tissue of the joints, leading to loss of function and mobility. It affects $1 \%$ of the population worldwide and about 4 million in China. RA is strongly prevalent in women (about $70 \%$ of sufferers are women) [77]. RA, in fact, responds to the definition of 'complex genetic disease'. A recent meta-analysis [78] conducted on Europeans has shown how complex the disease is by adding to the known susceptibility genes, 7 more, one of which is also shared by the Asian population [79]. These results have helped better characterize RA in the European and Asian population.

Challenges that lie ahead include the development of appropriate algorithms and statistics to handle all available omics data sets and integrate them into a systems perspective. Data integration will also help to clarify how existing therapies can be used in novel ways and dosages to counterbalance the side effects of conventional therapies.

Liu et al. have shown that to achieve this kind of integration, the translation of existing algorithms can be successful, for example, on metagenomic data [80], and that the integration of multi-omics data sets warrants a larger information base than the analysis of single layers. The use of multivariate statistics (such as factor analysis) to analyze jointly different types of omics data, for example mRNA and miRNA, was shown to successfully identify features that could not be found by the differential analysis of the mRNA and miRNA data sets separately $[81,82]$.

Other approaches rely on the flexibility of network representations, which allows more information than a list of molecules can provide, to be embedded in the algorithms. $\mathrm{Wu}$ et al. [83] have recently constructed a comprehensive RA map of all available transcriptional and post transcriptional information from omic screens (at the time mostly microarrays for gene expression). The map was integrated with information from known pathways to achieve a well interconnected map which could assist topological analysis. Interestingly, using this approach we were able to suggest a convincing explanation of the systemic effect of R406 inhibitor in the treatment of RA and to recommend novel contraindications, in particular, for the translation of diabetes therapies to RA patients (Nardini et al., in preparation).

\section{Conclusion}

Different layers of omics data suggest that distinct molecular and regulatory signatures are involved in disease processes. By combining high-throughput sequencing methods with computational approaches, researchers can now deve- lop comprehensive atlases of sequence-based mechanisms together with detailed molecular signatures for further understanding human diseases in a systematic way. In clinical terms, findings can be applied to personal diagnosis and then personalized medicine.

1 Lander E S, Linton L M, Bruce B, et al. Initial sequencing and analysis of the human genome. Nature, 2001, 409: 860-921

2 Venter J C, Adams M D, Myers E W, et al. The sequence of the human genome. Science, 2001, 291: 1304-1351

3 Pennisi E. Breakthrough of the year. Human genetic variation. Science, 2007, 318: 1842-1843

4 Nielsen R. Genomics: in search of rare human variants. Nature, 2010, 467: 1050-1051

5 Nik-Zainal S, Alexandrov L B, Wedge D C, et al. Mutational processes molding the genomes of 21 breast cancers. Cell, 2012, 149: 979-993

6 Pasaniuc B, Rohland N, McLaren P J, et al. Extremely low-coverage sequencing and imputation increases power for genome-wide association studies. Nat Genet, 2012, 44: 631-635

7 Collins F. Has the revolution arrived? Nature, 2010, 464: 674-675

8 Servin B, Stephens M. Imputation-based analysis of association studies: candidate regions and quantitative traits. PLoS Genet, 2007, 3: e114

9 Ashley E A, Butte A J, Wheeler M T, et al. Clinical assessment incorporating a personal genome. Lancet, 2010, 375: 1525-1535

10 Bird A. Perceptions of epigenetics. Nature, 2007, 447: 396-398

11 Greer E L, Shi Y. Histone methylation: a dynamic mark in health, disease and inheritance. Nat Rev Genet, 2012, 13: 343-357

12 Chi P, Allis C D, Wang G G. Covalent histone modificationsmiswritten, misinterpreted and mis-erased in human cancers. Nat Rev Cancer, 2010, 10: 457-469

13 Mann B S, Johnson J R, Cohen M H, et al. FDA approval summary: vorinostat for treatment of advanced primary cutaneous T-cell lymphoma. Oncologist, 2007, 12: 1247-1252

14 Sekigawa I, Kawasaki M, Ogasawara H, et al. DNA methylation: its contribution to systemic lupus erythematosus. Clin Exp Med, 2006, 6: 99-106

15 Maciejewska-Rodrigues H, Karouzakis E, Strietholt S, et al. Epigenetics and rheumatoid arthritis: the role of SENP1 in the regulation of MMP-1 expression. J Autoimmun, 2010, 35: 15-22

16 Miao F, Smith D D, Zhang L, et al. Lymphocytes from patients with type 1 diabetes display a distinct profile of chromatin histone H3 lysine 9 dimethylation: an epigenetic study in diabetes. Diabetes, 2008, 57: 3189-3198

17 Amir R E, Van den Veyver I B, Wan M, et al. Rett syndrome is caused by mutations in X-linked MECP2, encoding methyl-CpG-binding protein 2. Nat Genet, 1999, 23: 185-188

18 Tsankova N, Renthal W, Kumar A, et al. Epigenetic regulation in psychiatric disorders. Nat Rev Neurosci, 2007, 8: 355-367

19 Barski A, Cuddapah S, Cui K, et al. High-resolution profiling of histone methylations in the human genome. Cell, 2007, 129: 823-837

20 Eckhardt F, Lewin J, Cortese R, et al. DNA methylation profiling of human chromosomes 6, 20 and 22. Nat Genet, 2006, 38: 1378-1385

21 Hesselberth J R, Chen X, Zhang Z, et al. Global mapping of protein-DNA interactions in vivo by digital genomic footprinting. Nat Methods, 2009, 6: 283-289

$22 \mathrm{Yu} \mathrm{H}$, Zhu S, Zhou B, et al. Inferring causal relationships among different histone modifications and gene expression. Genome Res, 2008, 18: 1314-1324

23 Xiao S, Xie D, Cao X, et al. Comparative epigenomic annotation of regulatory DNA. Cell, 2012, 149: 1381-1392

24 Jin C, Li J, Green C D, et al. Histone demethylase UTX-1 regulates $C$. elegans life span by targeting the insulin/IGF-1 signaling pathway. Cell Metab, 2011, 14: 161-172

25 Xue H, Xian B, Dong D, et al. A modular network model of aging. Mol Syst Biol, 2007, 3: 147 
26 Zhou B, Yang L, Li S, et al. Midlife gene expressions identify modulators of aging through dietary interventions. Proc Natl Acad Sci USA, 2012, 109: E1201-E1209

27 Wei G, Wei L, Zhu J, et al. Global mapping of H3K4me3 and H3K27me3 reveals specificity and plasticity in lineage fate determination of differentiating $\mathrm{CD}^{+}{ }^{+} \mathrm{T}$ cells. Immunity, 2009, 30: 155-167

28 Wei G, Abraham B J, Yaqi R, et al. Genome-wide analyses of transcription factor GATA3-mediated gene regulation in distinct $\mathrm{T}$ cell types. Immunity, 2011, 35: 299-311

29 Voineagu I, Wang X, Johnston P, et al. Transcriptomic analysis of autistic brain reveals convergent molecular pathology. Nature, 2011, 474: 380-384

30 Kang H J, Kawasawa Y I, Cheng F, et al. Spatio-temporal transcriptome of the human brain. Nature, 2011, 478: 483-489

31 Yang L, Duff M O, Graveley B R, et al. Genomewide characterization of non-polyadenylated RNAs. Genome Biol, 2011, 12: R16

32 Yin Q F, Yang L, Zhang Y, et al. Long noncoding RNAs with snoRNA ends. Mol Cell, 2012, 48: 219-230

33 Hvistendahl M. My microbiome and me. Science, 2012, 336: 1248-1250

34 Snyder M, Du J, Gerstein M. Personal genome sequencing: current approaches and challenges. Genes Dev, 2010, 24: 423-431

35 Chen R, Mias G I, Li-Pook-Than J, et al. Personal omics profiling reveals dynamic molecular and medical phenotypes. Cell, 2012, 148: 1293-1307

36 Davis B D. The isolation of biochemically deficient mutants of bacteria by means of penicillin. Proc Natl Acad Sci USA, 1949, 35: 1-10

37 Bedell M A, Jenkins N A, Copeland N G. Mouse models of human disease. Part I: techniques and resources for genetic analysis in mice. Genes Dev, 1997, 11: 1-10

38 Bedell M A, Largaespada D A, Jenkins N A, et al. Mouse models of human disease. Part II: recent progress and future directions. Genes Dev, 1997, 11: 11-43

39 Hardouin S N, Nagy A. Mouse models for human disease. Clin Genet, 2000, 57: 237-244

40 Francis-West P H, Robson L, Evans D J. Craniofacial development: the tissue and molecular interactions that control development of the head. Adv Anat Embryol Cell Biol, 2003, 169: III-VI, 1-138

41 Wilkie A O, Morriss-Kay G M. Genetics of craniofacial development and malformation. Nat Rev Genet, 2001, 2: 458-468

42 Allanson J E, O'Hara P, Farkas L G, et al. Anthropometric craniofacial pattern profiles in Down syndrome. Am J Med Genet, 1993, 47: 748-752

43 Allanson J E, Hennekam R C. Rubinstein-Taybi syndrome: objective evaluation of craniofacial structure. Am J Med Genet, 1997, 71: 414-419

44 Allanson J E, Cole T R. Sotos syndrome: evolution of facial phenotype subjective and objective assessment. Am J Med Genet, 1996, 65: 13-20

45 Allanson J E, Hall J G, Hughes H E, et al. Noonan syndrome: the changing phenotype. Am J Med Genet, 1985, 21: 507-514

46 Beaty T H, Murray J C, Marazita M L, et al. A genome-wide association study of cleft lip with and without cleft palate identifies risk variants near MAFB and ABCA4. Nat Genet, 2010, 42: 525-529

47 Birnbaum S, Ludwig K U, Reutter H, et al. Key susceptibility locus for nonsyndromic cleft lip with or without cleft palate on chromosome 8q24. Nat Genet, 2009, 41: 473-477

48 Mangold E, Ludwig K U, Birnbaum S, et al. Genome-wide association study identifies two susceptibility loci for nonsyndromic cleft lip with or without cleft palate. Nat Genet, 2010, 42: 24-26

49 Rahimov F, Marazita M L, Visel A, et al. Disruption of an AP-2alpha binding site in an IRF6 enhancer is associated with cleft lip. Nat Genet, 2008, 40: 1341-1347

50 Boehringer S, van der Lijn F, Liu F, et al. Genetic determination of human facial morphology: links between cleft-lips and normal variation. Eur J Hum Genet, 2011, 19: 1192-1197

51 Kayser M, de Knijff P. Improving human forensics through advances in genetics, genomics and molecular biology. Nat Rev Genet, 2011, 12: $179-192$
52 Little A C, Jones B C, DeBruine L M. Facial attractiveness: evolutionary based research. Philos Trans R Soc Lond B Biol Sci, 2011, 366: 1638-1659

53 Meyer-Marcotty P, Alpers G W, Gerdes A B, et al. Impact of facial asymmetry in visual perception: a 3-dimensional data analysis. Am J Orthod Dentofacial Orthop, 2010, 137: 168 e1-e8; discussion 168-169

54 Albert A M, Ricanek K Jr, Patterson E. A review of the literature on the aging adult skull and face: implications for forensic science research and applications. Forensic Sci Int, 2007, 172: 1-9

55 Fu Y, Guo G, Huang T S. Age synthesis and estimation via faces: a survey. Pattern Analysis and Machine Intelligence, IEEE Transactions on, 2010, 32: 1955-1976

56 Bowyer K W, Chang K, Flynn P. A survey of approaches and challenges in $3 \mathrm{D}$ and multi-modal $3 \mathrm{D}+2 \mathrm{D}$ face recognition. Comput $\mathrm{Vis}$ Image Und, 2006, 101: 1-15

57 Burton A M, Wilson S, Cowan M, et al. Face recognition in poor-quality video: evidence from security surveillance. Psychol Sci, 1999, 10: 243-248

58 Poh M Z, McDuff D J, Picard R W. Non-contact, automated cardiac pulse measurements using video imaging and blind source separation. Opt Express, 2010, 18: 10762-10774

59 Boehringer S, van der Lijin F, Liu F, et al. Genetic determination of human facial morphology: links between cleft-lips and normal variation. Eur J Hum Genet, 2011, 19: 1192-1197

60 Farkas L G, Katic M J, Forrest C R. International anthropometric study of facial morphology in various ethnic groups/races. J Craniofac Surg, 2005, 16: 615

61 Weinberg S M, Naidoo S D, Bardi K M, et al. Face shape of unaffected parents with cleft affected offspring: combining threedimensional surface imaging and geometric morphometrics. Orthod Craniofac Res, 2009, 12: 271-281

62 Hammond P, Hutton T J, Allanson J E, et al. 3D analysis of facial morphology. Am J Med Genet A, 2004, 126: 339-348

63 Maal $\mathrm{T}$ J, van Looon B, Plooij J M, et al. Registration of 3-dimensional facial photographs for clinical use. J Oral Maxillofac Surg, 2010, 68: 2391-2401

64 Wan J, Shen L, Fang S, et al. A framework for 3D analysis of facial morphology in fetal alcohol syndrome. In: Proceedings of the 5th International Conference on Medical Imaging and Augmented Reality, 2010. 118-127

65 Guo J, Mei X, Tang K. Automatic landmark annotation and dense correspondence registration for 3D human face images. arXiv: 1212.4920

66 Bhuiyan Z A, Klein M, Hammond P, et al. Genotype-phenotype correlations of 39 patients with Cornelia De Lange syndrome: the Dutch experience. J Med Genet, 2006, 43: 568-575

67 Hammond P, Hannes F, Suttie M, et al. Fine-grained facial phenotype-genotype analysis in Wolf-Hirschhorn syndrome. Eur J Hum Genet, 2012, 20: 33-40

68 Hammond P, Hutton T J, Allanson J, et al. 3D dense surface models identify the most discriminating facial features in dysmorphic syndromes. In: 54th Annual Meeting of the American Society for Human Genetics, Toronto, Canada, 2004. 1

69 Hutton T J, Buxton B F, Hammond P, et al. Estimating average growth trajectories in shape-space using kernel smoothing. IEEE Trans Med Imag, 2003, 22: 747-753

70 Hammond P, Hutton T J, Allanson J E, et al. Discriminating power of localized three-dimensional facial morphology. Am J Hum Genet, 2005, 77: 999-1010

71 Hammond P, Forster-Gibson C, Chudley A E, et al. Face-brain asymmetry in autism spectrum disorders. Mol psychiatry, 2008, 13: 614-623

72 Kasperavičiūtė D, Catarino C B, Chinthapalli K, et al. Uncovering genomic causes of co-morbidity in epilepsy: gene-driven phenotypic characterization of rare microdeletions. PLoS ONE, 2011, 6: e23182

73 Han J D. Understanding biological functions through molecular networks. Cell Res, 2008, 18: 224-237 
74 Yeger-Lotem E, Riva L, Su L J, et al. Bridging high-throughput genetic and transcriptional data reveals cellular responses to alpha-synuclein toxicity. Nat Genet, 2009, 41: 316-323

75 Chen F, Zhang W, Liang Y, et al. Transcriptome and network changes in climbers at extreme altitudes. PLoS ONE, 2012, 7: e31645

76 Huang J, Liu Y, Zhang W, et al. eResponseNet: a package prioritizing candidate disease genes through cellular pathways. Bioinformatics, 2011, 27: 2319-2320

77 Scher J U, Abramson S B. The microbiome and rheumatoid arthritis. Nat Rev Rheumatol, 2011, 7: 569-578

78 Stahl E A, Raychaudhuri S, Remmers E F, et al. Genome-wide association study meta-analysis identifies seven new rheumatoid arthritis risk loci. Nat Genet, 2010, 42: 508-514

79 Kochi Y, Okada Y, Suzuki A, et al. A regulatory variant in CCR6 is associated with rheumatoid arthritis susceptibility. Nat Genet, 2010, 42: 515-519

80 Liu Y, Zhang C, Zhao L, et al. Adapting functional genomic tools to metagenomic analyses: investigating the role of gut bacteria in relation to obesity. Brief Funct Genomics, 2010, 9: 355-361

81 Fronza R, Tramonti M, Atchley W R, et al. Joint analysis of transcriptional and post-transcriptional brain tumor data: searching for emergent properties of cellular systems. BMC Bioinformatics, 2011, 12: 86

82 Fronza R, Tramonti M, Atchley W R, et al. Brain cancer prognosis: independent validation of a clinical bioinformatics approach. J Clin Bioinforma, 2012, 2: 2

83 Wu G, Zhu L, Dent J E, et al. A comprehensive molecular interaction map for rheumatoid arthritis. PLoS ONE, 2010, 5: e10137

Open Access This article is distributed under the terms of the Creative Commons Attribution License which permits any use, distribution, and reproduction in any medium, provided the original author(s) and source are credited. 